INPLASY

PROTOCOL

To cite: Zhao et al. Effects of SGLT2is on cardiovascular death and all-cause death in patients with diabetic

nephropathy: a meta-analysis of randomized controlled trials. Inplasy protocol 202120023. doi:

10.37766/inplasy2021.2.0023

Received: 06 February 2021

Published: 06 February 2021

Corresponding author:

Mei Qiu

13798214835@sina.cn

Author Affiliation:

Shenzhen Longhua District

Central Hospital

Support: None.

Review Stage at time of this submission: Preliminary searches.

Conflicts of interest:

None.

\section{Effects of SGLT2is on cardiovascular death and all-cause death in patients with diabetic nephropathy: a meta-analysis of randomized controlled trials}

Review question / Objective: No studies already published are powered to assess the effects of sodium-glucose cotransporter 2 inhibitors (SGLT2is) on cardiovascular death (CVD) and all-cause death (ACD) in patients with type 2 diabetes (T2D) and chronic kidney disease (CKD).

Condition being studied: This meta-analysis will assess the effects of SGLT2is on various cardiorenal outcomes mainly including CVD and ACD among patients with T2D and CKD. Information sources: We will systematically search Embase and PubMed using pre-planned search strategies, for identifying relevant cardiovascular or renal outcome trials.

INPLASY registration number: This protocol was registered with the International Platform of Registered Systematic Review and Meta-Analysis Protocols (INPLASY) on 06 February 2021 and was last updated on 06 February 2021 (registration number INPLASY202120023).

\section{INTRODUCTION}

Review question / Objective: No studies already published are powered to assess the effects of sodium-glucose cotransporter 2 inhibitors (SGLT2is) on cardiovascular death (CVD) and all-cause death (ACD) in patients with type 2 diabetes (T2D) and chronic kidney disease (CKD).

Condition being studied: This metaanalysis will assess the effects of SGLT2is on various cardiorenal outcomes mainly including CVD and ACD among patients with T2D and CKD. 


\section{METHODS}

Search strategy: It is being improved.

Participant or population: Adults with type 2 diabetes (T2D) and chronic kidney disease (CKD).

Intervention: Any SGLT2i. We will not consider the dosage of drugs as an effect modifier.

\section{Comparator: Placebo.}

Study designs to be included: Randomized controlled trials (RCTs) aiming to assess cardiovascular or renal outcomes.

Eligibility criteria: As shown in the above PICOS criteria.

Information sources: We will systematically search Embase and PubMed using preplanned search strategies, for identifying relevant cardiovascular or renal outcome trials.

Main outcome(s): 1. CVD. 2. ACD.

Additional outcome(s): 1. Major adverse cardiovascular events (MACE), defined as a composite of CVD, nonfatal myocardial infarction (MI), or nonfatal stroke. 2. Fatal and nonfatal MI. 3. Fatal and nonfatal stroke. 4. CVD or hospitalization for heart failure (HHF). 5. HHF. 6. Kidney function progression (KFP), i.e., a renal composite outcome, defined as a composite of sustained $40 \%$ reduction in estimated glomerular filtration rate (eGFR) or doubling of serum creatinine, end-stage kidney disease (ESKD) or initiation of renalreplacement therapy, or renal death. If this composite outcome is not available, we will use other one which is similar with this one instead.

Data management: The articles identified by the retrieval of two online databases will be assessed for relevance according to their titles and abstracts, and then those potentially eligible studies will be assessed for the final eligibility according to the inclusion and exclusion criteria. Two authors will then independently extract prespecified data from the included studies using a standardized Excel data extraction sheet. The pre-specified data to be extracted contain study type, key characteristics of included trials and participants, type of intervention, type of control, study outcomes. Any disagreements relevant with study selection and data extraction will be resolved through discussion with a third author. Treatment effects will be presented as hazard ratios (HRs) and $95 \%$ confidence intervals (Cls).

Quality assessment / Risk of bias analysis: Two authors will independently use the Cochrane risk of bias assessment tool to assess the risk of bias for included RCTs. Any disagreements related to risk of bias assessment will be resolved through discussion with a third author.

Strategy of data synthesis: We will use hazard ratios (HRs) and 95\% confidence intervals $(\mathrm{Cls})$ as reported in original studies to perform meta-analysis. We will measure statistical heterogeneity by calculating 12 statistic. If $\mathbf{I} \leq 50 \%$, metaanalysis will be done with fixed-effects model. Otherwise, meta-analysis will be done with random-effects model. $P<0.05$ or the low limit of $95 \%$ Cls of HRs $>1.0$ or the upper limit of those $<1.0$ denotes statistical significance. We will implement all statistical analyses in the Stata/MP software (version 16.0).

\section{Subgroup analysis: Not preplanned.}

Sensitivity analysis: Not preplanned.

Country(ies) involved: China.

Keywords: SGLT2is, type 2 diabetes, chronic kidney disease, cardiovascular death, all-cause death.

Contributions of each author:

Author 1 - Li-Min Zhao.

Author 2 - Liang-Liang Ding.

Author 3 - Ze-Lin Zhan.

Author 4 - Mei Qiu. 